\title{
European Bioanalysis Forum recommendation on method establishment and bioanalysis of biomarkers in support of drug development
}

\begin{abstract}
Biomarkers have become increasingly important in drug development and many bioanalysts are getting involved. Consequently, different views on how to approach the bioanalysis of biomarkers have been published or are being developed. The European Bioanalysis Forum has intensively discussed this topic since 2010 and is ready with their recommendation on method establishment and bioanalysis of biomarkers. Acknowledging that the challenges step outside the bioanalytical laboratory is a cornerstone of our recommendation. The importance of integrating all scientific aspects, from purely analytical aspects, all the way to understanding the biology and effects of the biomarker, prior to embarking on method establishment or sample analysis, cannot be underestimated. Close and iterative interactions with the teams requesting the data is imperative to develop a bioanalytical strategy that combines science, analytical performance and regulations. The European Bioanalysis Forum developed a straightforward decision tree to help the scientific community in developing a bioanalytical strategy for any biomarker in drug development.
\end{abstract}

\section{Introduction \& scope}

In this manuscript, the European Bioanalysis Forum (EBF) reports back from their internal discussions on the method establishment and bioanalysis of biomarkers in support of drug development performed in the regulated bioanalytical environment. Initially, these discussions were an integral part of an EBF subteam assigned to provide a recommendation on the practical implementation of the tiered approach principles. This subteam started their activities in 2008 , following up on the publication of the Crystal City III proceedings [1] and have already reported back on a first deliverable: application of the tiered approach applied in the quantification of metabolites in relation to the Metabolites in Safety Testing guideline [2]. In 2010, the EBF wanted to give priority to a recommendation in light of the ongoing Metabolites in Safety Testing discussions after the publication of the related regulatory guidances $[101,102]$.

From 2010 onwards, an EBF biomarker team was formed out of the aforementioned tiered approach team to further investigate how the EBF can contribute to the already intense discussions on biomarker bioanalysis within the global bioanalytical community. We acknowledged the many interesting and important technical papers and White Papers already published on the bioanalysis of biomarkers. Certainly, articles such as the 'fit-for-purpose' paper impacted the (bio)analytical community's approach to biomarker bioanalysis [3]. Nevertheless, although the latter paper provides excellent insight into the science of how to approach biomarker bioanalysis, the EBF experienced that the industry was moving forward too often to analyze biomarkers using existing regulated bioanalysis standards [4,103-105] or remained confused on fully embracing the opportunities and tiered approach of these 'fit-for-purpose' principles. Consequently, the EBF Biomarker team, consisting of bioanalytical experts from both pharmaceutical companies and CROs, identified the need to contribute to this discussion by integrating the internal EBF knowledge and reflections on the tiered approach with the already existing practices applied for the bioanalysis of a biomarker into an EBF recommendation.

As part of our recommendation and publication strategy, preludes of the insights in this manuscript were already shared for input and socializing at the 4th Open Symposium in Barcelona, Spain [5].

Although all our discussions intended to refer to biomarker analysis requests entering the traditional regulated bioanalysis laboratory, irrespective of size of the molecule of analytical technique involved, the principles of the recommendations we propose in this manuscript may also apply for other areas such as diagnostics or commercial immunoassay kits or similar. We did

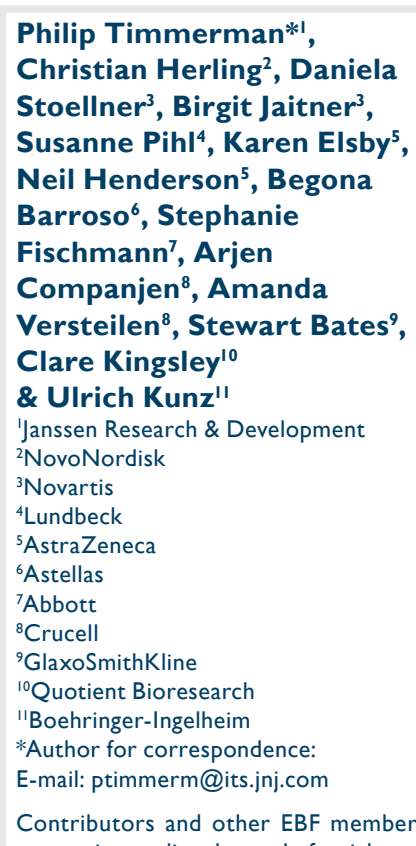
companies are listed at end of article. 
not consider this during our discussions, so we leave it up to the experts in the respective fields of diagnostics to decide how our recommendation adds value to their specific environment.

\section{The practical challenge of biomarkers: a few examples}

As an illustration, we present two hypothetical, but very recognizable, situations of inadequate strategies of biomarker analysis, both illustrating the need for crossfunctional communication and smart experimental design of the in-life part of the study as well as the bioanalytical strategy.

For both examples we reflect on the usefulness, or lack thereof, of the concentration data after the bioanalytical laboratory established the assays in line with the acceptance criteria on accuracy and precision as required by regulated bioanalysis guidelines. For clarity, in this manuscript 'method establishment' refers to the combined process of method development followed by method qualification or method validation of the assay in the context of bioanalytical science. As a bioanalytical community, we are mindful that validation may have a different meaning in the context of biomarker development.

In the first example, the assay showed a poor performance after initial method establishment, and the back-calculated values for all QC samples were barely within $50-150 \%$ of their nominal values. Additional method optimization took considerable time and effort to adjust the assay and bring it within improved acceptance criteria on accuracy and precision. Unfortunately, the team requesting the biomarker concentrations (in the continuation of this manuscript referred to as 'investigator' and assumed to be able to provide full disclosure of the science and purpose of the data generated, including statisticians), did not inform the bioanalyst that the anticipated pharmacodynamic (PD) effect of the drug on the biomarker would lead to a 20 - to 100 -fold increase of the concentrations. As a consequence, even though the initially developed assay had a poor performance, it would still have been capable of documenting the observed concentration changes. Insufficient information concerning the desired performance for the assay caused the bioanalytical team to waste significant resources in optimizing the assay.

A second example illustrates the opposite situation: the performance of the assay is according to requirements for regulated bioanalysis. The goal of the experiment was to document a $30 \%$ increase of the biomarker concentrations from basal in experimental animals. As a consequence of insufficient communication between the bioanalyst and the investigator, the experimental design of the in-life part of the study and the performance of the method was not aligned to provide solid answers. Indeed, although both individual and mean concentration versus time profiles suggest an increase in biomarker concentrations, by analyzing the individual samples from only six replicate animals, the assay was not able to unambiguously prove the desired effect. Upfront acknowledgment of the disconnect of the performance of the assay and the desired outcome of the study by the investigator and the bioanalytical scientist would have led to a more appropriate study design. Changes or enhancements could, for example, be to include more than six animals in the experiment or request replicate analysis of the samples from the original six animals. Even additional development and qualification/validation of the assay towards an accuracy and precision that excels the acceptance criteria of regulated bioanalysis could have been considered as an option.

Both examples perfectly illustrate the need to fully understand the purpose of the experiment. Insufficient information on this purpose and subsequent inappropriate use of established and broadly applied acceptance criteria as defined in regulated bioanalysis can lead to under- or over-performing assays. While an overperforming assay might lead to inappropriate use of resources, data generated by an underperforming assay could lead to misinterpretation of data used for making important decisions regarding drug safety or efficacy. FIGURE I shows a graphical illustration of both examples.

\section{EBF reflections on biomarker classification}

It is generally known that biomarkers are a very broad class of compounds and their analysis involves multiple and diverse bioanalytical competencies, disciplines and platforms [6-10,106]. Hence, a simple and straightforward 'one-sizefits-all' classification of biomarkers may be difficult if not impossible to design. Within the EBF, we looked at a number of ways of classifying biomarkers with a focus on connecting the analytical strategy with the tiered approach and, as such, facilitating which process or assay performance to choose for their bioanalysis in a transparent and reproducible way. We identified four major systems to classify biomarkers in relation to their bioanalysis, none of which we 

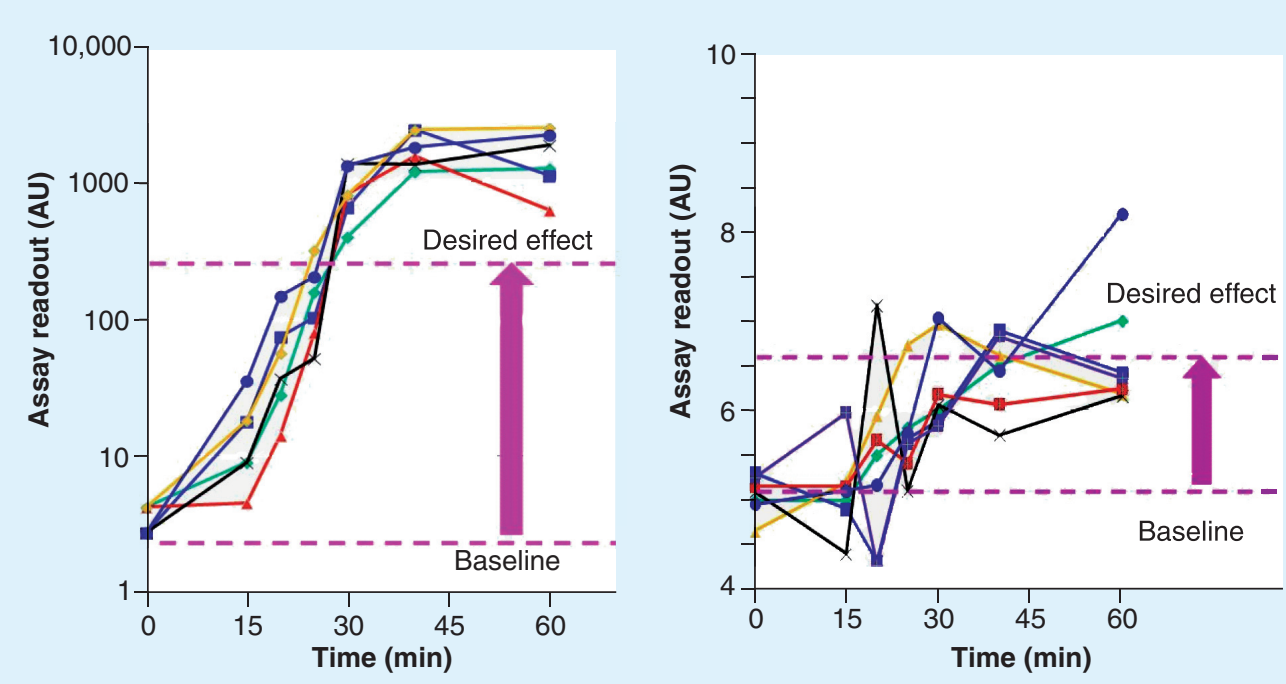

Figure 1. Examples of concentration versus time curves of two biomarkers.

propose to apply in isolation. Each classification starts from a different viewpoint and in order to make a good decision on the desired assay, we recommend to consider all four classification systems in concert.

\section{I. Observed or anticipated biomarker levels}

The first and most important consideration when establishing a biomarker assay is to understand the anticipated range of expression levels that the assay will need to cover and how expression will likely change under different conditions. This could include expression changes in response to drug treatment (PD biomarker), between healthy and pathological states (diagnostic biomarker) or within a disease or healthy population (predictive or prognostic biomarkers).

Oversimplified, the effect of the drug or condition exerted on the biomarker is 2D: biomarker concentrations increase (upregulation) or decrease (downregulation), both having more nuances:

- Upregulation of the biomarker concentrations with major (logarithmic/exponential) concentration changes;

- Upregulation of the biomarker concentrations with minor concentration changes;

- Downregulation of the biomarker concentrations with major (logarithmic/exponential) concentration changes;

- Downregulation of the biomarker concentrations with minor concentration changes.
In practice, the situation will, of course, be more complex: typical concentration versus time/condition curves of a biomarker may be a mixture of the scenarios mentioned above. In addition, these concentration changes can come on top of natural circadian concentration fluctuations.

In essence, the recommendation from this first classification system is to ensure the existing (or to be established) assay is able to document the anticipated concentration changes accurately and reproducibly. This is obviously not a new insight. However, in practice if we do not consider the anticipated concentration changes and just apply general bioanalytical run acceptance criteria, the assay potentially runs the risk of being over- or under-qualified, as illustrated in the examples presented in section 2.

A graphical visualization of PD markers as part of the first classification system is presented in FIGURE 2.

\subsection{Development phase in which a} biomarker is measured

Mistakenly, the development phase in which a biomarker is measured is often a major driver to decide on the level of validation (or qualification) needed for the assay. In fact, a biomarker measured in support of the drug-discovery phase often invites the bioanalytical laboratory to only focus upon the scientific deliverable of the assay and spend less time on method development establishment; whereas, the need to generate accurate and reproducible concentration data may be more critical for compound selection in 


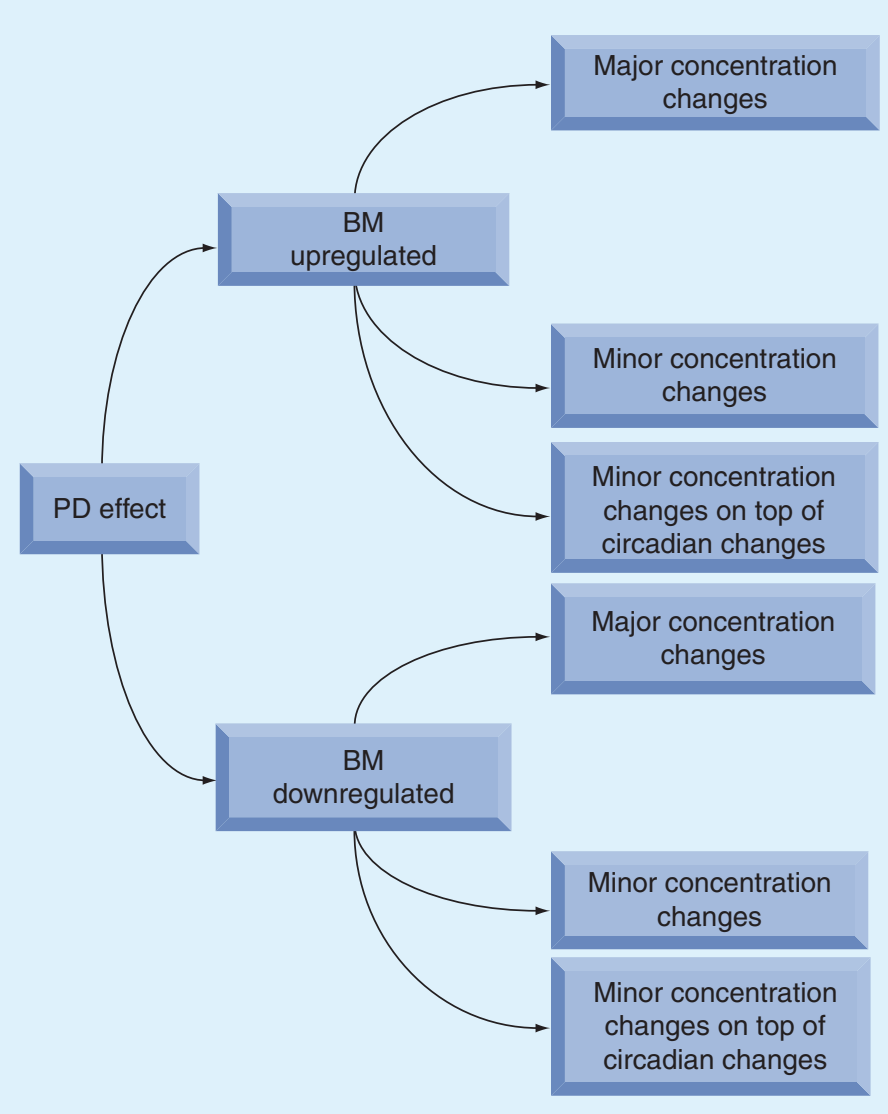

Figure 2. Range of expression changes of pharmacodynamic markers. BM: Biomarker; PD: Pharmacodynamics.

this early stage of drug development. Similarly, biomarkers measured in later stages of drug development triggers the laboratory to increase the focus on aspects of compliance, resulting in method validation strategies in line with regulated bioanalysis standards where this may not be needed at all. In our discussions, we concluded that the development phase in which a biomarker is measured can give some additional guidance if used appropriately and may contribute to the decision on the desired performance of the assay. However, we recommend not to apply this second classification system in isolation. Even primary focus on adherence to regulated bioanalysis guidelines triggered by the development phase in which the biomarker is measured has limited added value and can hamper good bioanalysis.

\subsection{Decisions taken from the} biomarker data

Together with the observed or anticipated concentration changes described in 3.1, this third classification system is equally important. In line with current thinking in industry, the EBF strongly supports highlighting the importance of the decisions taken from the data, irrespective of the phase of drug development. In any phase of drug development, the concentration data can support a simple scientific question or (prepare for) a milestone decision on efficacy and safety of drugs in development. In support of this third classification system, the EBF developed a decision tree that combines the decisions taken from the biomarker data with the development phase in which the data are generated.

In our recommendation, we distinguished two scenarios:

- Biomarker analysis is in support of efficacy decisions: we recommend to focus on the scientific aspects of the biomarker assay and only add additional focus on compliance (i.e., perform method validation using regulated bioanalysis standards) for assays used for pivotal efficacy decisions in clinical development. For any other biomarker data in support of efficacy decisions generated in a preclinical or research setting, no adherence to regulated bioanalysis requirements are recommended.

- Biomarker analysis is in support of safety decisions: we recommend to ensure appropriate method validation, apply regulated bioanalysis standards and criteria but consider and decide a priori the scientific requirements of the assay in all phases of development. For the latter, we recommend to adapt acceptance criteria in accordance with the anticipated performance needed by the assay as part of method validation. Furthermore, we recommend a flexible approach to the application of regulated bioanalysis standards as elaborated in 5.1.4.

\subsection{Possible fit of the assay specifics with regulated bioanalysis guidelines}

Unfortunately, in some cases the assay performance and/or format may fit perfectly with the current guidelines on regulated bioanalysis. However, in this manuscript we deliberately use the semantics 'unfortunately', since the ease or closeness of fit of a biomarker assay with established regulated bioanalysis guidelines has an unexpected and even undesired result: the bioanalytical scientist may prefer the commodity offered by this fit over the more challenging recommendations proposed in section 3.1 and 3.3. The desired scientific performance of the assay may be at risk because the bioanalyst tries to squeeze the assay into their comfort 
zone: a format that fits a standard operating procedure. The EBF recommendation on this fourth classification system is very clear: use the benefit of a potential fit with regulated bioanalytical guidelines only if the aforementioned classification systems support this fit.

The EBF recognizes that more classification systems for biomarker bioanalysis can be identified.

The four classification systems are superimposable and should be applied together to tailor an individual bioanalytical strategy in support of a biomarker request. By combining all four classifications in a simple and smart way, the EBF has developed a tool to help the bioanalytical scientist during method establishment of a novel biomarker assay or when applying (or adapting) an existing method to answer any request for biomarker analysis.

\section{Inform \& be informed}

In order to apply the above classification system successfully, the EBF also focused their discussions on a fifth principle upon which the overall recommendation is built: ensure regular, crossfunctional and iterative communication with the investigator requesting the biomarker concentration data (e.g., the pharmacologist, pharmacokineticist, toxicologist, clinician or others).

Often the geographical and/or scientific distance between the bioanalytical laboratory and investigator is a real and significant hurdle. The lack of efficient crossfunctional communication can lead to a situation whereby the bioanalytical laboratory decide upon assay requirements purely on its bioanalytical potential. In addition, insufficient understanding and subsequent inadequate communication from the investigator can push the bioanalytical laboratory in an undesired direction, leading to poor/overly developed methods and inappropriate use of resources. In addition to the examples in section 2, we all recognize the situation in which a pharmacologist reaches out to a bioanalytical laboratory requesting to validate an assay for a particular biomarker, but whereby both parties actually have a completely different understanding of what 'validated' means. The pharmacologist only wants the method to produce valid data to allow a good decision, whereas the bioanalyst may spend an additional few weeks generating a validated method to comply with regulated bioanalysis standards. The result is a perfect method that may not be able to provide the desired answer. In our recommendation (Figure 3), we put significant emphasis on the importance of regular and timely communication between the bioanalytical laboratory and the investigator, as an integral part of successful biomarker bioanalysis.

\section{Decision tree for biomarker method establishment \& analysis of study samples}

Based upon the above classification and subsequent reflections, $\mathrm{EBF}$ is proposing a decision tree to guide biomarker method establishment and the application of these assays for analysis of study samples. EBF refrained from trying to provide detailed scientific input, since the combination of 'biomarker assay-biomarker request' should be seen as a unique relationship requiring unique scientific answers. Nevertheless, we emphasize the importance to consider the science of the assay as the first and foremost important part of any bioanalytical strategy for biomarkers.

The decision tree should help the bioanalytical community to clearly define the level of scientific and regulatory validation needed (or not needed) for any biomarker request.

The decision tree starts from two discrete situations: first, request for analysis of biomarkers involving a novel assay and, second, a request for analysis of biomarkers using an established assay.

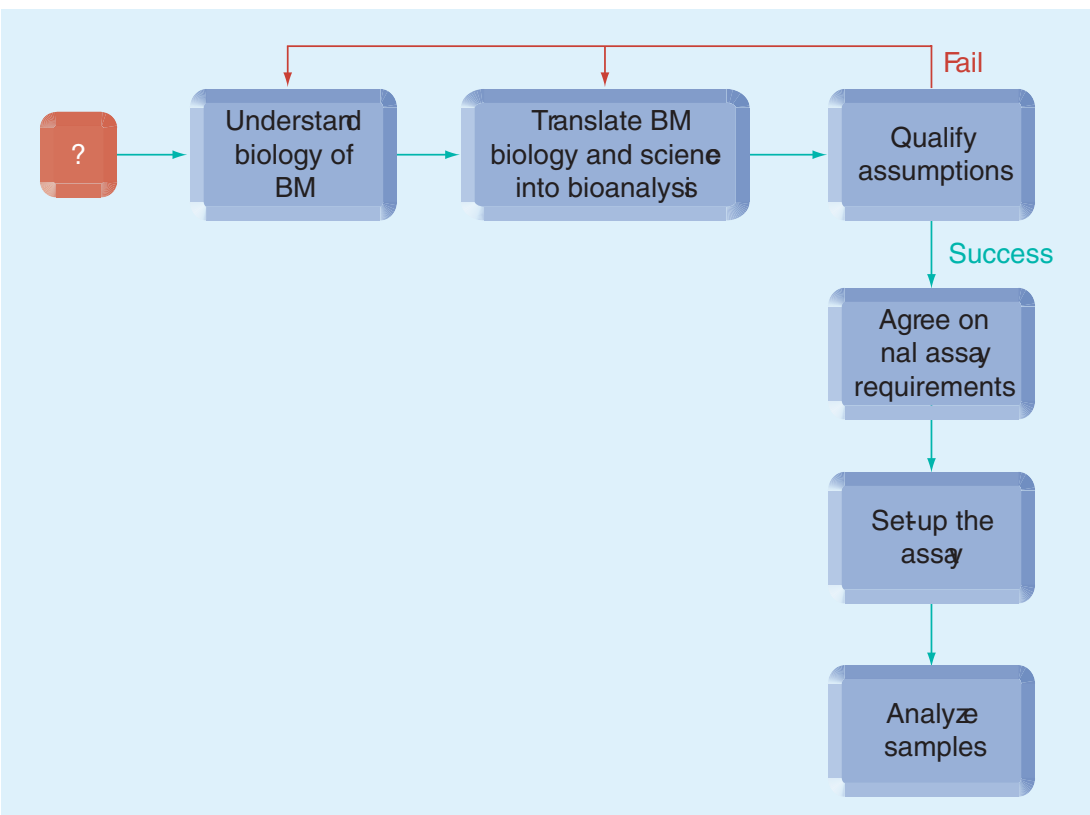

Figure 3. Decision flow for biomarker method establishment and sample analysis: new biomarker assay or platforms.

BM: Biomarker. 
In both situations, and once the scientific aspects of the biomarker itself are understood (e.g., biology, physicochemistry and so forth), our recommendation emphasizes the need to know the intended use of the data as the primary driver: PD, diagnostic or predictive/prognostic biomarkers? From that question, the EBF reflects and recommends a path forward to ensure valid data are generated in line with scientific and regulatory requirements. The recommendations should also help to manage the smart use of resources.

\section{I. Analysis of biomarkers using a novel assay}

The first part of the decision tree makes recommendations on biomarkers for which no assay is available at the time of the request. As part of the decision tree, the bioanalytical scientist will need to establish a new assay. The decision tree involves a sequence of steps to be taken.

\section{I.I. Understand the biology \& science of the biomarker}

In the first instance, the bioanalysis laboratory gets a request to perform bioanalysis of a biomarker in support of a (pre) clinical study at any stage of drug development. In practice, and as part of our recommendation, any request to perform bioanalysis should involve a discussion with the investigator. This discussion is essential to inform the bioanalytical scientist on the (anticipated) concentration changes of the biomarker, the intended target species and matrix and other important aspects of the request. This crucial information is needed in addition to the general scientific information on the chemistry and biology of the biomarkers mentioned in the introduction of this paragraph. We acknowledge that it is often difficult to get all the information on the table, but in this article we want to emphasize the importance of being adequately informed from the start. From here, the bioanalytical scientist needs to translate all the scientific information of the request (biology, physicochemistry, anticipated behavior or effect of the biomarker) into bioanalytical language: what is (are) the target analyte(s)? In which species/matrix do we need to measure? What is the anticipated concentration range? Are stability issues anticipated?

A second deliverable, often insufficiently appreciated, is to also define some language around the desired accuracy and precision of the assay. The desired features of the assay mentioned in the first deliverable are relatively easy to translate into assay requirements by any experienced bioanalyst. However, the second requirement, being an important cornerstone of the 'fit-for-purpose' principles, is more difficult to define and underappreciated by most bioanalytical scientists. Many laboratories fall back on their standard approaches on defining accuracy and precision as defined in regulated bioanalysis requirements. This can easily result in assays that are either too good (over-qualified) or not good enough (under-qualified), as illustrated in the examples from section 2. Nevertheless, referring to acceptance criteria applicable in regulated bioanalysis can be helpful and should be considered as a first option. Another consequence of this second deliverable requires the investigator to provide sufficient background on intended use of the data in relation to the stage of development, as well as decisions taken from that date moving forward. This should allow the bioanalyst and investigator to agree on the inclusion/exclusion of regulatory requirements in the bioanalytical strategy. Typically, for novel biomarker questions, one can assume that the data will be used for scientific assessments and familiarization of the biomarker, rather than immediately in support of safety or efficacy decisions. Consequently, the initial assay performance should focus on science and should not be hampered by regulatory requirements, with the potential for further validation at a later stage, should the assay require it. Finally, the discussions at this initial stage should also give the bioanalytical scientist the opportunity to inform the investigator of the 'ins and outs' of (regulated) bioanalysis and what is reasonable to expect and affordable to request.

As an illustration, a few examples of the deliverables from this first step are:

- Example 1: a biomarker is a safety marker. An assay is needed in human cerebrospinal fluid. Baseline concentrations of the biomarker in cerebrospinal fluid are a few $\mathrm{ng} / \mathrm{ml}$ with intrasubject circadian concentration changes of two to threefold from basal. (Expected) PD effect of the drug on the BM (= PK of the biomarker) results in an upregulation to fivefold of basal.

- Example 2: a method is required that can document plasma ratio changes of two biomarkers. Basal values of the biomarker 1/biomarker 2 ratio is 0.01 . The PD effect of the drug on the biomarker ratio is expected to experiences a twofold increase of biomarker 1/biomarker 2 ratio. In addition, both $\mathrm{BMs}$ can interconvert exvivo. 


\section{I.2. Translate biology \& science knowledge} into bioanalytical strategy

Based on the outcome of the above interactions, and after translation of all information into bioanalytical language, the laboratory will propose a bioanalytical strategy that should provide an adequate answer (i.e., accurate, precise, reproducible and robust) to the questions posed. Peer-reviewed literature is an important source of information to define this strategy.

The deliverable from this proposed bioanalytical strategy relates to the identification of technology and assay format, including practical aspects such as the preferred analytical platform (e.g., LC-MS cell-based, enzymatic or ligandbinding assay, or other), identification and availability of reference material for the target analytes(s) (i.e., authentic standards, certified reference standards, surrogate standards), identification and availability of (surrogate) matrix, and so forth. It is certainly recommended that some of this information/these materials are already available as part of the initial discussions with the investigator.

It is important to note that a validated assay for the biomarker may already be available, but this assay may not be suitable to provide the required answers to the specified questions (accuracy, precision, range and target matrix). In this case, the validated assay may serve as a basis from which the required assay is established.

\section{I.3. Preliminary testing of the proposed strategy}

Once the assay format and anticipated performance have been identified, the questions from 5.1.1 and proposed strategy from 5.1.2 are put into practice by developing a preliminary assay. The EBF proposes not to take assay development beyond a stage (or investment) where the assay can provide a reasonable answer to the questions defined in 5.1.1. If possible and available, the preliminary assay should be tested on real samples. In comparison to assays developed for new chemical entities and new biological entities (all exogenous compounds), biomarkers are developed for endogenous compounds. Therefore, any sample harvested in the correct manner and available for research purposes, such as method establishment, can be used for this.

The deliverable from this stage is a preliminary method tested on real samples. Based on the performance of this assay, we identified two scenarios:
- Preliminary method confirms proposals made in 5.I.2

In this scenario, the EBF suggests that the bioanalytical scientist reconnects with the investigator to report back on the performance of the preliminary method. The bioanalyst and the investigator should agree on the performance required for the final method, including the need for the assay and the data generated by the assay, to comply with regulatory requirements. The bioanalytical method can now be established, as further discussed in 5.1.4. At the same time and if applicable, the (pre)clinical protocol should refer to the biomarker and its required assay performance requirements. For biomarkers involving regulatory review, the investigator should consider including references to the biomarker measurements in the study protocol.

\section{- Preliminary method fails to meet the assumptions} in 5.I.I or the plan in 5.I.2

This scenario has many shades of grey. The bioanalytical scientist may have done or can do a number of method development iterations to optimize the assay towards meeting the anticipated requirements. In addition, the method may be performing as originally anticipated, but the original assumptions made as part of the deliverables from 5.1.1 were inaccurate. Consequently, at this point we recommend to reconnect the bioanalytical laboratory and the investigator and jointly fine-tune the request in view of outcome from the preliminary method testing. As a result, both parties may agree to redirect the work, rethink the biomarker question from scratch, reset the required deliverable from the assay or focus on further method optimizations, including a change of the assay platform (e.g., from MS-based to ligand-based or vice versa), intensive method development, and so forth. Considering the potential investments required, we recommend only to embark on these method optimizations after rediscussion with the investigator, so all can agree on a best way forward.

For novel biomarker assays in support of a preliminary scientific assessment or for studies in the very early stages of development, the method establishment activities may be paused or stopped at this stage, because data and assay performance suffices for internal decision making.

\section{I.4. Set up the assay \& apply to study samples}

Once full agreements are reached, the bioanalytical laboratory can now move forward and 
finalize method establishment.

When methods are established and applied in the analysis of study samples, they should be aligned with current practices for screening, qualified and validated assays. For all tiers above screening methods, the EBF recommends a priori set acceptance criteria as much as is feasible. These a priori set acceptance criteria may even include a rationale on purpose and scope of the PK or PD behavior of the biomarker related to the initial request. The reason for the latter is the earlier reflection that the assay requirements for one specific biomarker may be different, depending on the PD effect we want to document. For example, assay requirements for estradiol determinations applied in postmenopausal subjects aiming to document a tenfold downregulation from low $\mathrm{pg} / \mathrm{ml}$ basal levels may be different from an assay in premenopausal subjects aiming to document a similar downregulation starting from ten- to 100-times higher basal levels. Inevitably, requirements of the assay on accuracy and precision, LLOQ, and potentially other parameters, are different.

It will be important during method establishment to define what qualified or validated means, with respect to acceptance criteria and regulatory requirements in the context of biomarker bioanalysis. Citing the EBF recommendations [5]:

- Screening methods are methods or procedures applied to samples for which no a priori acceptance criteria are available. The outcome may range from qualitative or relative response, or estimated abundance and can be used for early decision making;

- Qualified methods are methods with appropriate level of scientific validation. When applied to study samples, they generate absolute concentration data that allow documented and reproducible decision making;

- Validated methods are methods that follow the procedure around method development and validation as described in the regulatory Guidance on Bioanalytical Methods Validation. Applying this to biomarkers, additional flexibility may be needed for validated methods.

As part of our discussions, we recognize that for qualified or validated methods, absolute concentrations are not a prerequisite. An assay can also be established to provide qualified/validated results on biomarker ratios or a titer. As part of our discussions, we recognized that for qualified or validated methods, absolute concentrations are not a prerequisite in the context of biomarker analysis. An assay can also be established to provide qualified/validated results on biomarker ratios or a titer. Hence, the interpretation of screening, qualified and validated assays (as mentioned in [5]) applied in the context of biomarkers, should be broadened to allow this. For screening and qualified assays, the emphasis 'used for early decision making' and 'documented and reproducible decision making', respectively, will remain applicable.

Similarly, we suggest an (a priori) broadening of the acceptance criteria as defined for validated assay and support application thereof in the context of applying regulated bioanalysis for biomarker bioanalysis in the regulated space. As a consequence, the line between qualified and validated assay in the context of biomarkers may be less clear as for analysis of exogenous compounds, and careful considerations and good documentation will be needed to clarify this approach.

Our proposed method establishment process would look like this:

- Develop assay: in preparation of desired deliverable being either screening (e.g., in case of ratio testing), qualified or validated assays.

- Document assay performance in line with desired deliverable and including all required (and a priori defined) acceptance criteria. In case the assay format does not fit requirements described in current guidelines for regulated bioanalysis, but assay data are anticipated to conform with regulatory standards, it is advisable to predefine which parts of the assay cannot adhere to these guidelines and propose refined criteria. For example, wider or narrower acceptance criteria, choice of surrogate matrix, availability and certification of reference standards and so forth. This approach should be favored above trying to fit the assay into the standard regulated format or claiming regulatory status without documenting areas of noncompliance to these guidelines.

Finally, study samples can be analyzed. The decision whether or not to include additional postvalidation parameters like incurred sample reanalysis, selectivity testing towards concomitant drugs, or any specific stability testing was not discussed in the EBF Biomarker topic team. As for exogenous compounds, the EBF 
nevertheless feels that scientific considerations should supersede regulatory expectations. It should be noted that a thorough evaluation of the concentration data (preferably together with the investigator) and the assay performance during sample analysis is essential and may lead to refining the assay performance or redefining the assay requirements. We did not visualize this in the visualization of the overall recommendation for new biomarker platforms, presented in FIGURE 3.

\subsection{Bioanalysis of biomarkers using an existing assay}

In many cases, a biomarker method is already available and investigators simply submit the samples to the bioanalytical laboratory for analysis. However, there are multiple occasions where this approach may not be appropriate, for one simple reason: the available biomarker assay was not established for the intended purpose of the new request. Differences in the new request towards the available assay can range from matrix/species, concentration range or the LLOQ required, the biomarker being an isomer of the analyte of the existing assay and so forth. Consequently, we also recommend that a decision flow is followed for existing biomarker assays that is in line with the process recommended in 5.1. As can be anticipated, the decision flow for existing assays is even more simple than the latter (FIGURE 4).
5.2.I. Overlay performance of existing assay on biomarker request

Upon obtaining the request for analysis, the bioanalytical scientist is recommended to overlay the performance of the existing biomarker assay with the required or desired performance of the request. We also advise that the investigator is involved at some point in the process to ensure mutual understanding of the request.

\subsubsection{Agreement on final assay requirements}

The EBF identified two scenarios:

\section{The existing assay fits with the request}

Often biomarker data are needed within an ongoing project and can copy established and earlier agreed acceptance criteria. In this case, the bioanalytical laboratory runs the samples using the existing (screening, qualified or validated) method and following regulated bioanalysis standards as needed.

\section{The existing assay does not fit the request}

The request may have come in support of a different study design or a new project, and therefore requires different end points compared with the performance of the established assay. In this case, we recommend that the bioanalyst and the investigator jointly agree on the required enhancements to comply with scientific and/or regulatory needs

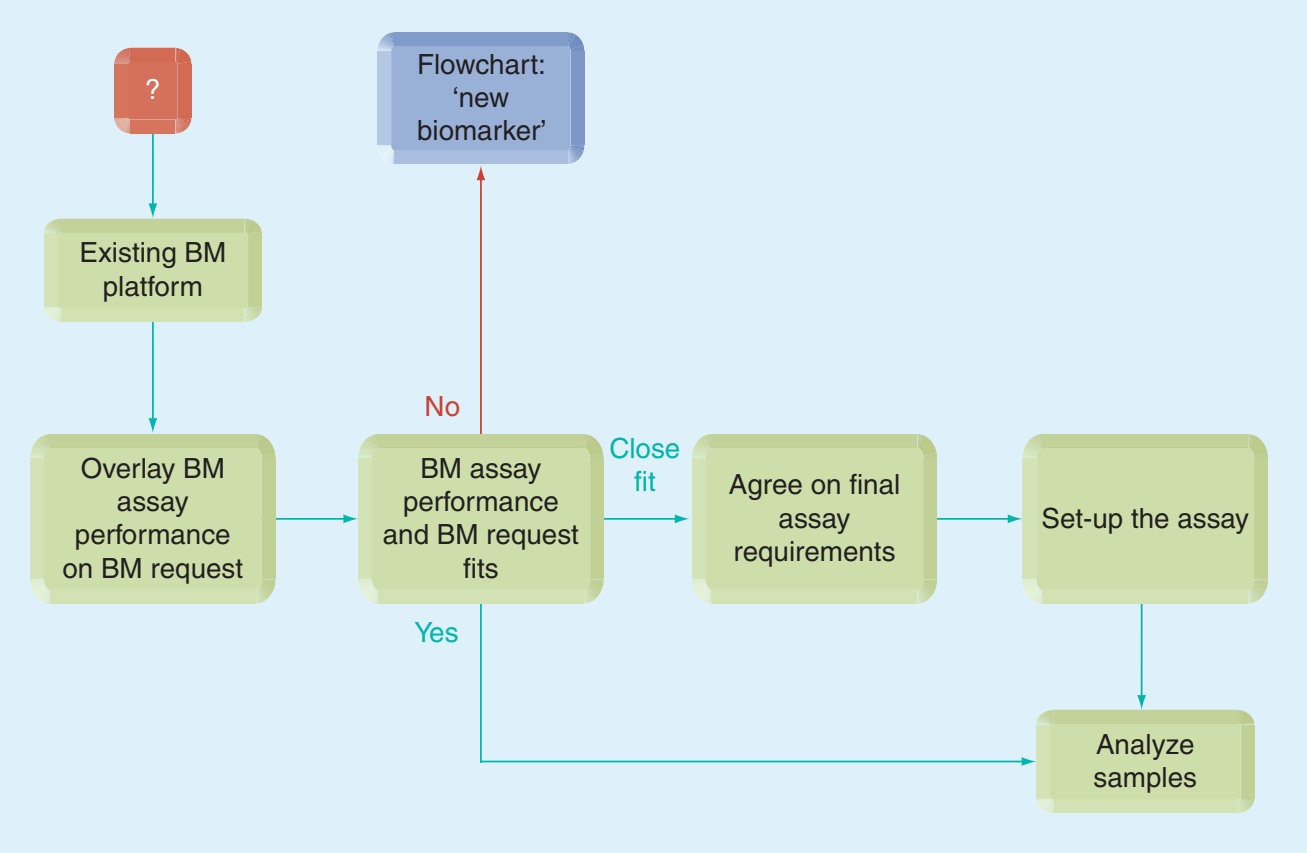

Figure 4. Decision flow for biomarker method establishment and sample analysis: existing biomarker assay or platforms.

BM: Biomarker. 
(i.e., optimize accuracy and precision, document additional selectivity, sensitivity, different matrix, anticoagulant and so forth) and follow the flowchart described in paragraph 5.1.1. Progressing through this flowchart will certainly not be as time- and resource-intensive as for novel biomarkers. Nevertheless, the assay changes needed may be significant to ensure a fit with the new request; for example, from qualified to validated, a more challenging matrix compared to the original one, a tenfold increase in sensitivity and so forth.

\subsubsection{Set up the assay \& apply to study} samples

As with 5.1.4., the assay is set up and the samples are analyzed, again in full agreement with the recommendations described in 5.1.4. Again, it should be noted that a thorough evaluation of the concentration data and assay performance during sample analysis is essential and may lead to refining the assay performance or redefining the assay requirements.

Both decision trees can be nicely combined into a single overall decision tree (FIGURE 5).

\section{Conclusion \& recommendation for biomarker bioanalysis}

The EBF recommends:

- Base any decision on biomarker assay requirements by considering the following four classifications: i Observed or anticipated concentration changes (PD effect);

ii Development phase in which the analysis is requested;

iii Decisions taken from the data;

iv Possible fit of the assay format with regulated bioanalysis guidelines.

- Evaluate the input from the above questions in concert to establish a strategy on the level of validation needed for the assay. We suggest priority is given to (i) and (iii), but recommend not to allow only one of the classification systems to decide on the performance of the assay.

- Ensure effective and iterative communication between the bioanalytical team and the investigator, to ensure mutual understanding and alignment of expectations of the assay performance prior to and during method establishment. It is recommended to do this even in those cases where an established method is available.

- Apply tiered approach principles when defining assay performance. In case assay performance needs to be in line with regulated bioanalysis requirements, allow and document flexible and scientific adaptation of these guidelines. Those

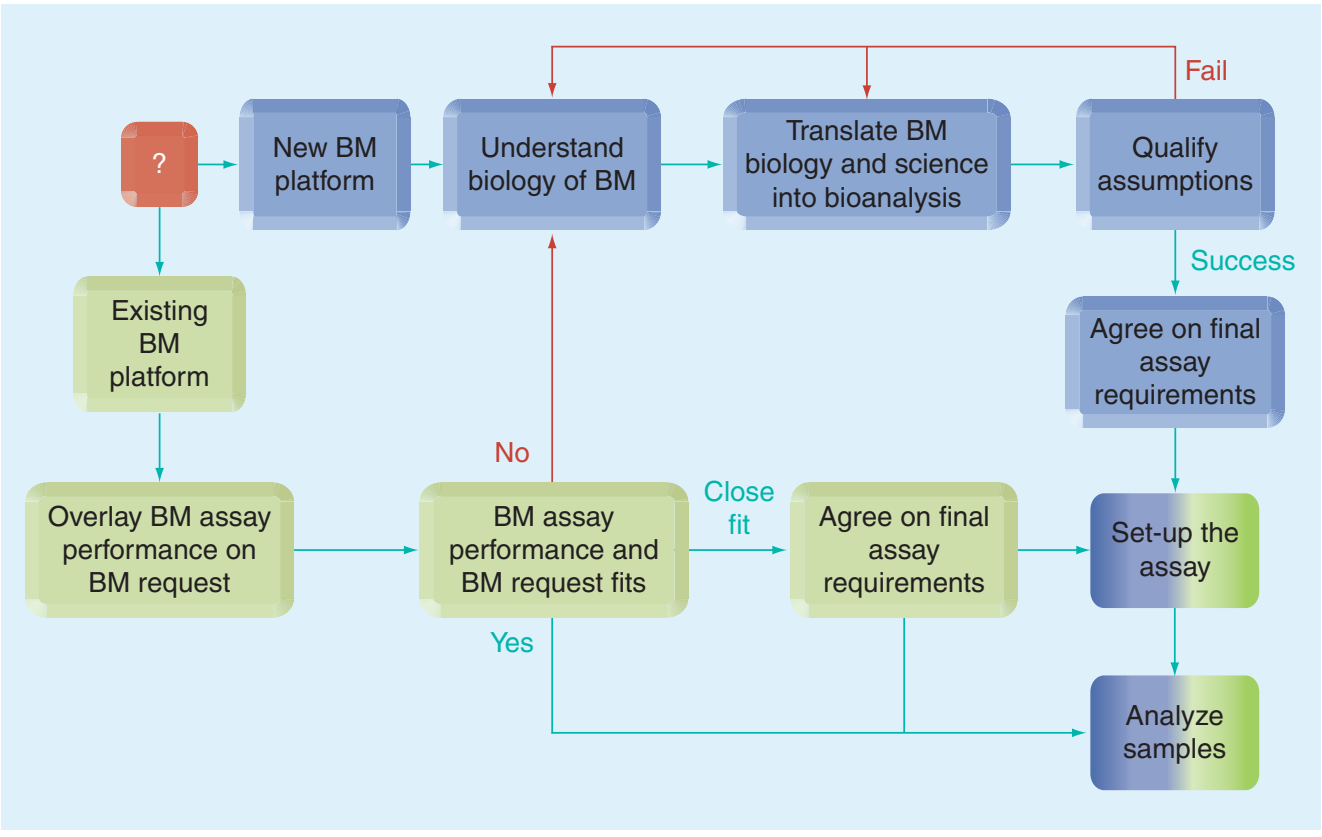

Figure 5. Decision flow for biomarker method establishment and sample analysis. BM: Biomarker. 
guidelines can be a solid basis to work from, but we recognize that they were not developed to manage the broad array of biomarkers.

- Apply a stepwise approach in developing an assay, starting from understanding the biology and science of the biomarker, confirming the scientific assumption made via a preliminary assay, prior to embarking on final method establishment and sample analysis.

\section{Financial \& competing interests disclosure}

The authors have no relevant affiliations or financial involvement with any organization or entity with a financial interest in or financial conflict with the subject matter or materials discussed in the manuscript. This includes employment, consultancies, honoraria, stock ownership or options, expert testimony, grants or patents received or pending, or royalties.

No writing assistance was utilized in the production of this manuscript.

Executive summary

- Make sure bioanalysts understand the scientific questions asked prior to method establishment or sample analysis.

Ensure continued communication between the bioanalytical team and the team requesting the data in order to align expectations on assay performance (scientific and regulatory).

Apply a stepwise approach when establishing an assay: consider starting with a preliminary assay to check initial scientific assumptions prior to embarking on final method establishment and sample analysis.

Apply principles of tiered approach when defining final assay performance, understanding the potential need to step out of the framework of regulated bioanalysis guidelines.

\section{Contributors \& other European Bioanalysis Forum member companies}

Peter van Amsterdam (Abbott Healthcare Products), Ingeborg Dreher (Abbott Laboratories), Mira Doig (ABS), Susanne Globig (Actelion), Carl-Johan Sennbro (Active Biotech), Josep Jansat (Almirall), Eva Vieser (Amgen), Hans Mulder (Astellas), Mathias Liljeblad (AstraZeneca), John Maltas (BASi), Margarete BrudnyKloeppel (Bayer), Bernhard Beckermann (Bayer), Silke Lüdtke (Boehringer-Ingelheim), Christine Schiebl (Celerion), Petra Struwe (Celerion), Stephen Williams (Charles River), Tim Sangster (Charles River Laboratories), Stuart McDougall (Covance), Ian Skitt (Covance), Berthold Lausecker (CRS), Uwe Wannenwetch (CRS), Yoshihisa Sano (Eisai), Rudi Segers (Eurofins), Edwin Janssen (Eurofins), Jordi Ortiz (Ferrer), Birgitte Buur Rasmussen (Ferring), Magnus Knutsson (Ferring), Jim Hillier (Gen-Probe), Klaus Pusecker (Grunenthal), Steve White (GlaxoSmithKline), Peter Sagelsdorff (Harlan), Irene Lentheric (Harlan), Alberto Guenzi (Hoffmann-La Roche), Eginhard Schick (Hoffmann-La Roche), John Smeraglia (Huntingdon Life Sciences), Joseph Marini (Janssen R\&D), Morten Anders Kall (Lundbeck), Achim Freisleben (Merck-Serono), Michaela Golob (Merck-Serono), Chris Cox (Millipore), Benno Ingelse (MSD), Nathalie Mokrzycki (MSD), Fernando Romero (Novartis), Marianne Scheel Fjording (NovoNordisk), Sirpa Laakso (Orion Pharma), Christian Kikuta (pharm-analyt Labor), Hermann Mascher (pharmanalyt Labor), Edwin Hooijschuur (PRA), Barry van der strate (PRA), Jaap Wieling (QPS), Rachel Green (Quotient Bioresearch), Dietmar Schmidt (Sanofi), Marie-Helene Pascual (Sanofi), Pascal Delrat (Servier), Bruno Le Bras (Servier), Richard Abbott (Shire), Christoph Siethoff (Swiss Bioquant), Winfried Redeker (SwissBioanalytics), Brigitte Buscher (TNO Triskelion), Jasja Wolthoorn (TNO Triskelion), Olivia Vetterlein (UCB), Ludovicus Staelens (UCB), Tammy Harter (Unilabs), Collin Farrel (Unilabs).

\section{References}

1 Viswanathan CT, Bansal S, Booth B et al. Workshop/Conference Report - quantitative bioanalytical methods validation and implementation: best practices for chromatographic and ligand binding assays. AAPS J. 9(1), E30-E42 (2007).

2 Timmerman P, Kall M, Gordon B et al. Best practices in a tiered approach to metabolite quantification: views and recommendations of the European Bioanalysis Forum. Bioanalysis 2(7), 1185-1194 (2010).

3 Lee J, Devanarayan V, Barrett Y et al. Fit-for-purpose method development and validation for successful biomarker measurement. Pharm. Res. 23(2), 312-328 (2006).

4 DeSilva B, Smith W, Weiner R et al. Recommendations for the bioanalytical method Validation of ligand-binding assays to support pharmacokinetic assessments of macromolecules. Pharm. Res. 20(11), 1885-1900 (2003).

5 Dijksman J, Timmerman P, Abbott R et al. 'Less is more': defining modern bioanalysis. Bioanalysis 4(6), 633-642 (2012).

6 Houghton R, Horro Pita C, Ward I et al. Generic approach to validation of smallmolecule LC-MS/MS biomarker assays. Bioanalysis 1(8), 1365-1374 (2009).

7 Biomarker Definitions Working Group. Biomarkers and surrogate end points: preferred definitions and conceptual framework. Clin. Pharmacol. Ther. 69, 89-95 (2001).

8 Danhof M, Alvan G, Dahl SG, Kuhlmann J, Paintaud G. et al. Mechanism-based pharmacokinetic-pharmacodynamic modeling - a new classification of biomarkers. Pharm. Res. 22(9), 1432-1437 (2005).
9 Lesko L, Atkinson A. Use of biomarkers and surrogate end points in drug development and regulatory decision making. Annu. Rev. Pharmacol. Toxicol. 41, 347-366 (2001).

10 Kuhlmann J. The Applications of Biomarkers in Early Clinical Drug Development to Improve Decision-Making Processes. In: Appropriate dose selectionHow to optimize clinical drug development. Venitz J, Sittner W (Eds). Springer, Berlin, Germany, 29-45 (2007).

\section{- Websites}

101 US Department of Health and Human Services, FDA, Center for Drug Evaluation and Research (2008). US FDA Guidance for Industry. Safety testing of drug metabolites. www.fda.gov/downloads/Drugs/ GuidanceCompliance RegulatoryInformation/Guidances/ ucm079266.pdf

102 ICH Topic M 3 (R2). Non-clinical safety studies for the conduct of human clinical trials and marketing authorization for pharmaceuticals (CPMP/ICH/286/95). www.emea.europa.eu/docs/en_GB/ document_library/Scientific_ guideline/2009/09/WC500002720.pdf

103 US Department of Health and Human Services, US FDA, Center for Drug Evaluation and Research, Center for Veterinary Medicine. Guidance for Industry: Bioanalytical Method Validation (2001). www.fda.gov/downloads/Drugs/ GuidanceComplianceRegulatory Information/Guidances/ucm070107.pdf 
104 European Medicines Agency. Guideline on bioanalytical method validation (2011). www.ema.europa.eu/docs/en_GB/ document_library/Scientific_ guideline/2011/08/WC500109686.pdf
105 Agência Nacional de Vigilância Sanitária. Guide for validation of analytical and bioanalytical methods (2003).

www.europeanbioanalysisforum.eu/files// Brazil\%20BA\%20Validation.pdf
106 Center for Drug Evaluation and Research. Guidance for Industry: qualification process for drug development tools (2010). www.c-path.org/pdf/FDADraftDDTools.pdf 